\title{
ATIVIDADES ANTIOXIDANTE, ANTIMICROBIANA E COMPOSTOS FENÓLICOS DE EXTRATOS COMERCIAL E IN NATURA DE CURCUMA LONGA
}

\author{
Antioxidant and Antimicrobial Activities and Phenolic Compounds of \\ Commercial and In Natura Extracts of Curcuma longa
}

Ana Luiza Lira'; Josieli Fátima Vesoloski²; Marcieli Peruzzolo³, Débora Zamprogna Flôres ${ }^{4}$ Rogério L. Cansian ${ }^{5}$, Natalia Paroul ${ }^{6}$

\begin{abstract}
${ }^{1}$ Graduada do Curso de Medicina Veterinária do Instituto de Desenvolvimento Educacional do Alto Uruguai. Mestranda do Curso de Engenharia de Alimentos da Universidade Regional Integrada do Alto Uruguai e das Missões - URI Erechim.E-mail: analuizalira8@gmail.com

${ }^{2}$ Graduada do Curso de Agronomia. Mestranda do Curso de Engenharia de Alimentos da Universidade Regional Integrada do Alto Uruguai e das Missões - URI Erechim.E-mail: josy_vesoloski@hotmail. com

${ }^{3}$ Graduada do Curso de Química Industrial. Mestranda do Curso de Engenharia de Alimentos da Universidade Regional Integrada do Alto Uruguai e das Missões - URI Erechim. E-mail: marciperuzzolo@yahoo.com.br

${ }^{4}$ Graduada do Curso de Engenharia de Alimentos. Mestranda do Curso de Engenharia de Alimentos da Universidade Regional Integrada do Alto Uruguai e das Missões - URI Erechim. E-mail: floresdeboraz@gmail.com

${ }^{5}$ Professor do Programa de Pós-Graduação Stricto sensu em Engenharia de Alimentos. Universidade Regional Integrada do Alto Uruguai e das Missões - URI Erechim. E-mail: cansian@uricer.edu.br

${ }^{6}$ Professora do Programa de Pós-Graduação Stricto sensu em Engenharia de Alimentos. Universidade Regional Integrada do Alto Uruguai e das Missões - URI Erechim.E-mail: nparoul@uricer.edu.br
\end{abstract}

Data do recebimento: 03/12/2020 - Data do aceite: 22/02/2021

RESUMO: A cúrcuma (Curcuma longa) é usada como aditivo e corante na indústria de alimentos, por possuir excelentes propriedades biológicas. O objetivo do trabalho foi comparar as atividades antioxidante, antimicrobiana e teor de compostos fenólicos totais dos extratos obtidos de cúrcuma em pó (comercializada) e do rizoma da cúrcuma in natura. A extração foi feita em Soxhlet utilizando etanol $95 \%$ por um período de seis horas e o rendimento obtido foi 
5,25 e 3,42\% de massa para o extrato de cúrcuma em pó e rizoma da cúrcuma in natura, respectivamente. A atividade antioxidante foi avaliada pelo método de captura de radicais livres (DPPH'), o teor de compostos fenólicos totais pelo teste de Folin-Ciocalteu. A concentração inibitória mínima dos extratos foi determinada para quatro bactérias, sendo duas Gram-positivas (Listeria monocytogenes e Staphylococcus aureus) e duas Gram-negativas (Salmonella choleraesuis e Escherichia coli). O extrato em pó apresentou maior teor de compostos fenólicos totais $\left(30,4 \mathrm{mg}\right.$ EAG/100g) e menor $\mathrm{IC}_{50}(0,068 \mathrm{mg} / \mathrm{mL})$ quando comparando com o extrato do rizoma in natura $(28,0 \mathrm{mg}$ EAG/100g e

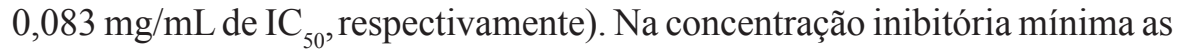
duas amostras mostram resultados satisfatórios para Salmonella choleraesuis e o extrato da cúrcuma em pó para Staphylococcus aureus.

Palavras-chave: Curcuma longa. Atividade antioxidante. Fenóis totais. Concentração inibitória mínima.

ABSTRACT: Turmeric (Curcuma longa) is widely used as food additive and colorant in the food industry, as it has excellent biological properties. The objective of this work was to compare the antioxidant, antimicrobial and total phenolic compounds content of extracts obtained from turmeric powder (commercialized) and from the natural turmeric rhizome. The extraction was carried out through Soxhlet extraction using $95 \%$ ethanol for a period of six hours and the yield obtained was 5,25 and 3,42\% of mass for the turmeric powder extract and turmeric rhizome in natura, respectively. The antioxidant activity was evaluated by the DPPH radical scavenging method, the content of total phenolic compounds by the Folin-Ciocalteu test. The minimum inhibitory concentration of the extracts was determined for four bacteria, two Gram-positive (Listeria monocytogenes and Staphylococcus aureus) and two Gram-negative (Salmonella choleraesuis and Escherichia coli). The powder extract presented a higher content of total phenolic compounds (30.4 mg EAG / 100g) and a lower IC50 (0.068 mg / mL) when compared with the extract of the rhizome in natura $(28.0 \mathrm{mg}$ EAG / $100 \mathrm{~g}$ and $0.083 \mathrm{mg} / \mathrm{mL}$ of IC50, respectively). At the minimum inhibitory concentration, both samples show satisfactory results for Salmonella choleraesuis and turmeric powder extract for Staphylococcus aureus.

Keywords: Curcuma longa. Antioxidant activity. Total phenols. Minimum inhibitory concentration.

\section{Introdução}

A cúrcuma (Curcuma longa L.), também conhecida como açafrão-da-terra, é uma planta pertencente à família Zingiberaceae, originária da Ásia. Foi introduzida no Brasil na década de 1980 e hoje é cultivada em diversas regiões. É usada como aditivo, pois possui propriedades antioxidantes e antimicrobianas e sua principal utilização é como corante e condimento na indústria alimentícia brasileira (OLIVEIRA et al., 2019). 
Existe uma tendência muito expressiva por parte da indústria e dos consumidores em reduzir o uso de aditivos químicos em diversos setores, inclusive na produção de alimentos. Dessa forma, a busca por soluções e alternativas mais naturais para manter a estabilidade microbiana e garantir a qualidade do produto têm crescido muito nos últimos anos (CRUZ; PEREIRA, 2010). Os aditivos são adicionados aos alimentos há muitos anos, e sua utilização visa a impedir alterações no que diz respeito a conservação do alimento, melhorar seu aroma, cor, sabor sem prejudicar seu estado nutritivo. A extração de aditivos naturais tem se mostrado muito eficiente no que diz respeito a esses parâmetros, conferindo grande potencial antioxidante e antimicrobiano (OLIVEIRA et al., 2019).

Segundo Ballen (2019), apesar dos microrganismos serem mais conhecidos como agentes deteriorantes, o processo de oxidação também diminui o tempo de prateleira e a qualidade dos alimentos e, nesse caso, o agente mais comum é o oxigênio, aliado à falta de técnicas de processamento adequadas ao produto.

De acordo com a definição de Halliwell e Gutteridge (2007), antioxidante é "qualquer molécula que atrasa, previne ou remove um dano oxidativo de uma molécula alvo". Essas biomoléculas podem ser protegidas através dos antioxidantes que podem atuar de diferentes formas: impedindo a formação dos radicais livres, inibindo a oxidação do substrato, interceptando os radicais livres gerados pelo metabolismo através da ação enzimática ou pelos agentes de sacrifício, o qual tem a função de proteger essas biomoléculas.

Também segundo Ballen et al. (2019), a maioria dos antioxidantes utilizados hoje pelas indústrias ainda são sintéticos (que podem se tornar carcinogênicos se utilizados em doses inadequadas) e por essa razão cresce cada vez mais o número de estudos voltados a substituição destes por naturais.
Entretanto, a estabilidade ao armazenamento de antioxidantes naturais pode ser afetada e existe uma lacuna na literatura comparando a atividade antioxidante e antimicrobiana de extratos em pó comerciais e extrato do rizoma in natura de cúrcuma.

Diante do exposto, o objetivo do estudo foi comparar as atividades antioxidantes, antimicrobianas e avaliar teor de compostos fenólicos totais dos extratos alcoólicos obtidos a partir de cúrcuma em pó e rizoma de cúrcuma in natura.

\section{Materiais e Métodos}

\section{Material biológico}

A cúrcuma em pó foi obtida no comércio local, embalada e distribuída por Linea Verde Alimentos Ltda de Curitiba-PR e os rizomas foram obtidos numa propriedade rural de Barão de Cotegipe-RS no mês de outubro de 2020. Estas últimas foram higienizadas com água corrente e armazenadas ao abrigo da luz até o momento da extração, onde foram raladas em ralador convencional de uso doméstico, sendo que o tamanho das partículas não foi determinado para ambas amostras.

\section{Obtenção dos Extratos}

As extrações foram realizadas em equipamento Soxhlet com $200 \mathrm{~mL}$ de etanol 95\% (Química moderna), em triplicata, segundo a metodologia IAL (2008), adaptada. Foram usados $10 \mathrm{~g}$ de cúrcuma em pó e $10 \mathrm{~g}$ do rizoma ralado. $\mathrm{O}$ sistema foi mantido em refluxo por de 6 horas. Os extratos foram concentrados em estufa à $60^{\circ} \mathrm{C}$ até atingir massa constante.

O rendimento foi expresso em porcentagem, conforme a Equação 1.

$$
R_{E}(\%)=\frac{\left(M_{2}-M_{1}\right)}{M_{A}} 100
$$


Onde:

$R_{E}(\%)$ - Rendimento em porcentagem; $M_{2}$ - Massa do extrato + balão; $M_{1}$ - Massa do balão; $M_{A}$ - Massa da amostra de cúrcuma

\section{Atividade Antioxidante}

A metodologia é fundamentada na medida da extinção da absorção do radical 2,2-difenil-1-picril hidrazil ( $\mathrm{DPPH}^{\circ}$ ) em $515 \mathrm{~nm}$, por método espectrofotométrico (SILVESTRI et al., 2010).

Os extratos foram diluídos em etanol em diferentes concentrações $(0,0025$ a $0,75 \mathrm{mg} /$ $\mathrm{mL}$ ). A análise foi realizada misturando 500 $\mu \mathrm{L}$ de amostra diluída e $500 \mu \mathrm{L}$ de solução 0,1 mmol.L ${ }^{-1}$ de DPPH ${ }^{*}$. A mistura, após ser agitada em agitador magnético (K40-10208) permaneceu em repouso por 30 minutos em temperatura ambiente e ao abrigo da luz. A leitura da absorbância foi feita em um espectrofotômetro UV-visível (UV-1600 Spectrophotometer) com comprimento de onda de $515 \mathrm{~nm}$.

A solução controle foi obtida substituindo-se $500 \mu \mathrm{L}$ da amostra por $500 \mu \mathrm{L}$ de álcool etílico. Para a solução denominada "branco" foi utilizada uma mistura extrato - álcool etílico sem DPPH. A atividade de captura de radicais pelos extratos é expressa como percentual de inibição de DPPH e calculada através da Equação (2):

$\mathrm{AA} \%=100-\left\{\left[\left(\mathrm{Abs}_{\text {amostra }}-\mathrm{Abs}_{\text {branco }}\right) \times 100\right] \div \mathrm{Abs}_{\text {controle }}\right\}$

Após a avaliação da faixa de concentração ideal, calculou-se a concentração de extrato de cúrcuma necessária para capturar 50\% do radical livre DPPH $\left(\mathrm{IC}_{50}\right)$ por análise de regressão linear.

\section{Fenóis Totais}

Os compostos fenólicos totais foram quantificados pelo teste de Folin-Ciocalteu, descrito por Singleton e Rossi (1965) com adaptações, em triplicata, utilizando ácido gálico como padrão. Foram misturados 0,5 $\mathrm{mL}$ de amostra (concentração de $0,1 \mathrm{mg} / \mathrm{mL}$ ) com 2,5 mL de reagente de Folin-Ciocalteau ( $1: 10 \mathrm{v} / \mathrm{v}$ em água) e 2,0 mL de solução de $\mathrm{Na}-$ ${ }_{2} \mathrm{CO}_{3}$ a $4 \%$. Os tubos foram homogeneizados e guardados ao abrigo da luz por duas horas. Após esse período foi feita a leitura de absorbância a $760 \mathrm{~nm}$ em um espectrofotômetro UV-visível (UV-1600 Spectrophotometer).

Para calcular o teor dos fenóis totais contido nas amostras, utilizou-se uma curva padrão de ácido gálico (GAE), com base em uma curva de calibração com concentrações de ácido gálico que variaram de 0 a 120 $\mu \mathrm{g} . \mathrm{mL}^{-1}$.

\section{Atividade Antimicrobiana}

Para os testes de concentração inibitória mínima (CIM) do extrato etanólico da cúrcuma em pó (CP) e o extrato do rizoma da cúrcuma in natura (CR), foram selecionados quatro microrganismos, duas bactérias Gram -positivas Listeria monocytogenes (ATCC 7644) e Staphylococcus aureus (ATCC 25923), e duas Gram-negativas Salmonella choleraesuis (ATCC 10708) e Escherichia coli (ATCC 25922, obtidas na American Type Culture Collection.

As cepas foram inoculadas e crescidas previamente em meio Luria Bertani (LB) (10 g.L $\mathrm{L}^{-1}$ de triptona, 5 g... $\mathrm{L}^{-1}$ de extrato de levedura e 5 g. $\mathrm{L}^{-1} \mathrm{de} \mathrm{NaCl}$ ) durante 24 horas a $36 \pm 1{ }^{\circ} \mathrm{C}$ em estufa bacteriológica.

Os extratos da cúrcuma foram diluídos em etanol em diferentes concentrações $(0,3906$ a $75 \mathrm{mg} / \mathrm{mL}$ ) e inoculados com $10 \mu \mathrm{L}$ de bactéria previamente crescida $\left(10^{8} \mathrm{UFC} / \mathrm{mL}\right)$. $\mathrm{O}$ etanol puro foi utilizado como controle 
para verificar o crescimento bacteriano. As leituras das amostras foram realizadas em leitor de microplacas (Biotek Instruments EL 800) a $490 \mathrm{~nm}$ em 0 hora e depois de 24 horas de incubação à $36 \pm 1{ }^{\circ} \mathrm{C}$ em estufa bacteriológica. A concentração inibitória mínima (CIM) foi definida como a menor concentração do extrato em $\mathrm{mg} / \mathrm{mL}$, capaz de inibir o crescimento microbiano, avaliado pela variação na densidade ótica da leitura final menos leitura inicial.

\section{Resultados e Discussões}

Os rendimentos dos extratos obtidos da cúrcuma em pó (CP) e do rizoma da cúrcuma in natura (CR) foram 5,25 e 3,42\%, respectivamente. Gomes (2018) alcançou rendimento de 17,34\%, Camatari (2017) de 15\% e Braga et al. (2003) tiveram rendimentos de $27 \%$, todos para extratos obtidos da cúrcuma em pó através de extração com Soxhlet em etanol. Gomes (2018) cita que essa diferença pode estar relacionada ao fato de o material vegetal do rizoma ter alto teor de umidade, mudanças climáticas nos diferentes cultivos, idade dos rizomas e tamanho das partículas maiores quando comparada com a cúrcuma em pó.

As Tabelas I e II apresentam os resultados obtidos da avaliação da atividade antioxidante dos fenóis totais dos extratos.

Tanvir et al. (2017) avaliaram duas variedades de cúrcuma, fazendo quatro experimentos diferentes em etanol, com amostras vindas de localidades distintas, e em seus melhores resultados obtiveram um $\mathrm{IC}_{50}$ de
0,00119 mg/mL. Akter et al. (2019), comparando a atividade antioxidante de diferentes espécies e variedades de cúrcuma, observaram atividade sequestrante de radical DPPH, significativamente maior (IC50 0,0264 mg/ $\mathrm{mL}$ ), na variedade Ryudai gold de Curcuma longa.

Os resultados das atividades antioxidantes encontradas corroboram com os autores citados anteriormente, pois demonstram uma ação antioxidante muito boa deste condimento. Os valores encontrados de 0,068 e de $0,083 \mathrm{mg} / \mathrm{mL}$ para cúrcuma em pó e rizoma, respectivamente, embora mais altos, mostram o potencial antioxidante desta espécie e, principalmente, que o extrato em pó de cúrcuma não perde sua atividade antioxidante em relação a cúrcuma imediatamente extraída. Essa diferença entre trabalhos pode estar ligada, além da idade das amostras, clima, extração, variedade e também pela localidade do cultivo, visto que todas as plantas e cultivares possuem suas condições ótimas para desenvolvimento em diferentes regiões, o que pode afetar em suas propriedades.

$\mathrm{O}$ extrato da cúrcuma em pó (CP) apresentou maior atividade antioxidante $\left(\mathrm{IC}_{50}\right.$ $0,068 \mathrm{mg} / \mathrm{g}$ ) e maior teor de compostos fenólicos totais $(30,4 \mathrm{mgEAG} / \mathrm{g}$ extrato) quando comparado com extrato obtido do rizoma in natura $\left(\mathrm{IC}_{50} 0,083 \mathrm{mg} / \mathrm{mL}\right)$ e fenólicos totais $(28,0 \mathrm{mgEAG} / \mathrm{g}$ extrato). Os resultados de atividade antioxidante estão diretamente relacionados com dos resultados de compostos fenólicos totais, pois os compostos fenólicos, que são formados por um ou mais anéis aromáticos e com pelo menos

Tabela I - Atividade antioxidante dos extratos alcoólicos da cúrcuma em pó e rizoma da cúrcuma in natura

\begin{tabular}{cccc}
\hline Extratos & $\mathrm{IC}_{50}(\mathrm{mg} / \mathrm{mL})$ & Equação da reta & $\mathrm{R}^{2}$ \\
\hline $\mathrm{CP}$ & 0,068 & $\mathrm{y}=559,62 \mathrm{x}+3,669$ & 0,98 \\
$\mathrm{CR}$ & 0,083 & $\mathrm{y}=282,08 \mathrm{x}+10,415$ & 0,93 \\
\hline
\end{tabular}

$\mathrm{CP}$ - extrato da cúrcuma em pó; $\mathrm{CR}$ - extrato do rizoma da cúrcuma in natura 
Tabela II - Teor de Fenóis Totais dos extratos alcoólicos da cúrcuma em pó e do rizoma da cúrcuma in natura

\begin{tabular}{cccr}
\hline Extratos & $\begin{array}{c}\text { Fenóis totais } \\
(\mathrm{mgEAG} / \mathrm{g} \text { de extrato })\end{array}$ & Equação da reta & $\mathrm{R}^{2}$ \\
\hline $\mathrm{CP}$ & 30,4 & $\mathrm{y}=0,0068 \mathrm{x}+0,028$ & 0,99 \\
$\mathrm{CR}$ & 28,0 & & \\
\hline
\end{tabular}

$\mathrm{CP}$ - extrato da cúrcuma em pó; $\mathrm{CR}$ - extrato do rizoma da cúrcuma in natura.

um grupo hidroxila, em geral, podem reagir com radicais livres, devido à facilidade com que o átomo de hidrogênio do grupo hidroxila pode ser separado por um radical livre.

SINGH et al. (2012) avaliaram os teores de diferentes substâncias em folhas, rizomas maduros e rizomas imaturos de C. longa e o teor de fenólicos totais de 27,42 $\mathrm{mgEAG} / \mathrm{g}$. Já Gomes (2018) obteve 196 e 20 mgEAG/g para os extratos em etanol e em hexano, respectivamente, sendo que o valor obtido para o extrato em etanol mostra que $19,6 \%$ do extrato equivale à ácido gálico. A elevada concentração de compostos fenólicos encontrada no respectivo trabalho é explicada por Gomes (2018). Ele cita que isso é uma característica do extrato alcoólico, pois estes compostos são mais solúveis em etanol, já que possuem em sua estrutura um ou mais grupos $\mathrm{OH}$, representando polaridade intermediária.
Na Tabela III é possível analisar a concentração inibitória para Escherichia coli, Salmonella choleraesuis, Staphylococcus aureus e Listeria monocytogenes

Observa-se que tanto o extrato em pó como o extrato do rizoma in natura de cúrcuma apresentaram concentração inibitória mínima inferior a do controle (etanol) sobre Salmonella choleraesuis, indicando atividade antibacteriana, principalmente para o extrato do rizoma da cúrcuma in natura. Para $S$. aureus, apenas o extrato em pó apresentou concentração inibitória mínima inferior ao controle. Já, para L. monocytogenes e E. coli, nenhum dos extratos demonstrou atividade superior ao controle.

Péret-Almeida et al. (2008) também analisaram a atividade de antimicrobiana de extratos dos rizomas em pó de C. longa e não observaram atividade contra $S$. aureus, Bacillus subtilis, S. choleraesuis, E. coli,

Tabela III - Concentração inibitória mínima dos extratos alcoólicos da cúrcuma em pó e do rizoma da cúrcuma in natura para inibição das bactérias Gram-negativas e Gram-positivas.

\begin{tabular}{cccc}
\hline \multirow{2}{*}{ Bactéria } & \multicolumn{3}{c}{ Concentração inibitória $(\mathrm{mg} / \mathrm{mL})$} \\
\cline { 2 - 4 } & $\mathrm{CP}$ & $\mathrm{CR}$ & Controle \\
\hline Gram-positivas & & & \\
Listeria monocytogenes & 4,687 & 4,687 & 3,125 \\
Staphylococcus aureus & 2,343 & 3,125 & 3,125 \\
\hline Gram-negativas & & & \\
\hline Salmonella choleraesuis & 4,687 & 2,343 & 6,250 \\
Escherichia coli & 4,687 & 4,687 & 3,125 \\
\hline
\end{tabular}

$\mathrm{CP}$ - extrato da cúrcuma em pó; $\mathrm{CR}$ - extrato do rizoma da cúrcuma in natura 
Aspergillus niger, Sacharomyces cerevisiae, e Candida albicans. Já o óleo essencial da cúrcuma obtido através de hidrodestilação, demonstrou a atividade antimicrobiana in vitro para o B. subtilis, S. choleraesuis, $E$. coli, $A$. niger e $S$. cerevisiae. Isto sugere que compostos com propriedades antimicrobianas presentes na cúrcuma são apolares, e os nossos extratos etanoicos possuem baixos teores dos mesmos.

Urbano (2017) não encontrou atividade antimicrobiana para as cepas Staphylococcus aureus e Escherichia coli. O autor complementa que isso pode ser indicativo de que o princípio ativo não foi suficiente ou que a interação entre os polímeros, a curcumina e o rizoma em pó não proporcionou um meio adequado para o princípio ativo agir como antimicrobiano.

\section{Conclusão}

Os extratos etanólicos da Curcuma longa apresentaram boa atividade antioxidante, sendo que o extrato da cúrcuma em pó mostrou maior rendimento, maior atividade antioxidante e maior teor de compostos fenólicos totais, indicando que a planta não perde suas propriedades durante o processamento e a estocagem. Tanto o extrato do pó da cúrcuma quanto o extrato do rizoma mostraram atividade para Salmonella choleraesuis e o extrato do pó para Staphylococcus aureus. Com os resultados obtidos observa-se que o extrato pode ser utilizado como um antioxidante natural.

\section{Agradecimentos}

Os autores agradecem ao Conselho Nacional de Desenvolvimento Científico e Tecnológico - Brasil (CNPq), a Coordenação de Aperfeiçoamento de Pessoal de Nível Superior - Brasil (CAPES) - Código Financeiro 001 e a Fundação de Amparo à Pesquisa do Estado do Rio Grande do Sul - Brasil (FAPERGS).

\section{REFERÊNCIAS}

AKTER, J.; HOSSAIN, M. A.; TAKATA, K.; ISLAM, M. Z.; HOU, D. X. Antioxidant activity of different species and varieties of turmeric (Curcuma spp): Isolation of active compounds.

Comparative Biochemistry and Physiology Part C: Toxicology \& Pharmacology, v. 215, p. 9-17, 2019.

BALLEN, S. C.; RIGO, D.; PALIGA, M.; PUTON, B. M. S.; CANSIAN, R. L.; PAROUL, N. Determinação do potencial antioxidante (DPPH) e antimicrobiano de extratos vegetais e óleo essencial de louro (Laurus nobilis). Perspectiva, n.163, v. 43, p. 61-70, 2019.

BRAGA, M. E. M.; LEAL, P. F.; CARVALHO, J. E.; M. MEIRELES, A. A. Comparison of yield, composition, and antioxidant activity of turmeric (Curcuma longa L.) extracts obtained using various techniques. Journal Agricultural Food Chemistry, v. 51, p. 6604-6611, 2003.

CAMATARI, F. O. S. Determinação de curcuminoides e avaliação da capacidade antioxidante contra espécies reativas de oxigênio e nitrogênio de extratos de Curcuma Longa e constituintes 
isolados. 2017. $149 \mathrm{f}$. Tese (Doutorado Química e Biotecnologia) - Universidade Federal de Alagoas, Maceió, 2017.

CRUZ, P. B., PEREIRA, C. A. M. Avaliação da presença de antimicrobianos naturais em condimentos industrializados. Revista Simbio-Logias, n. 5, v. 3, n.5, 2010.

GOMES, M. M. Obtenção e caracterização de extratos de Curcuma longa e aplicação na estabilidade oxidativa da manteiga. 2018. 28 f. Trabalho de Conclusão de Curso (Engenharia de Alimentos) - Universidade Federal da Fronteira Sul, Laranjeiras do Sul, PR, 2018.

HALliWELL, B.; GUTTERIDGE, J. M. C. Free Radicals in Biology and Medicine. 4. ed. Oxford: Clarendon Press, 2007.

IAL-Instituto Adolfo Lutz. Métodos físico-químicos para análise de alimentos. 4. ed. São Paulo, 2008.

OLIVEIRA, L. R. C.; DUTRA, R.; LACHNO, A. S.; OLIVEIRA, S. M.; SEVERO J. Bioativos e aditivos naturais em alimentos: Corantes, antioxidantes e aromatizantes. Boletim TécnicoCientífico, n. 2, v. 5, 2019.

PÉRET-ALMEIDA, L.; NAGHETINI, C. C.; NUNAN, E. A.; JUNQUEIRA, R. G.; GLÓRIA, M. B. A. Atividade antimicrobiana in vitro do rizoma em pó, dos pigmentos curcuminóides e dos óleos e dos essenciais da Curcuma longa L. Ciências Agrotécnicas, n. 3, v.32, p. 875-881, 2008.

SILVESTRI, J. D. F.; PAROUL, N.; CZYEWSKI, E.; LERIN, L.; ROTAVA, I.; CANSIAN, R. L.; MOSSI, A.; TONIAZZO, G.; OLIVEIRA, D.; TREICHEL, H. Perfil da composição química e atividades antibacteriana e antioxidante do óleo essencial do cravo-da-índia (Eugenia caryophyllata Thunb.). Revista Ceres, n. 5, v. 57, p. 589-594, 2010.

SINGH, P.; VISHWAKARMA, S. P.; SINGH, U.; SHUKLA, M.; SINGH, R.; SINGH, R. K.; SINGH, R. B.; WILSON, D. W.; SINGH, R. L. Quantification and Evaluation of Antioxidant Activity of Some Bioactive. The Open Nutraceuticals Journal, v. 5, p. 179-186, 2012.

SINGLETON, V. L.; ROSSI, J. A. Colorimetry of total phenolics with phosphomolybdicphosphotungstic acid reagents. American Journal of Enology and Viticulture, v. 16, p. 144-158, 1965.

TANVIR, E. M.; HOSSEN, M. D. S.; HOSSAIN, M D. F.; AFROZ, R.; GAN, S. H.; KHALIL, M. D. I. KARIM, N. Antioxidant Properties of Popular Turmeric (Curcuma longa) Varieties from Bangladesh. Journal of Food Quality, v. 2017, p. 8, 2017.

URBANO, H. P. Produção de filmes compostos de poliestireno com a adição de curcumina e rizoma em pó de Curcuma longa. 2017. 28 f. Trabalho de Conclusão de Curso (Engenharia de Alimentos) - Universidade Tecnológica Federal do Paraná, Campo Mourão, PR, 2017. 